\title{
Utilidad de las maniobras de reclutamiento (contra)
}

\author{
A. OCHAGAVIA, L. BLANCHY J. LÓPEZ-AGUILAR
}

Centro de Críticos. Hospital de Sabadell. Corporació Parc Taulí. CIBER Enfermedades Respiratorias. Instituto de Salud Carlos III. Institut Universitari Fundació Parc Taulí. Universitat Autònoma de Barcelona. Sabadell. Barcelona. España

En los pacientes con SDRA se observa una reducción del volumen pulmonar y el área de parénquima pulmonar útil para realizar el intercambio de gases. La aplicación de un patrón ventilatorio adecuado que incluya la aplicación de PEEP puede restituir el aireamiento de zonas pulmonares colapsadas y garantizar una distribución más homogénea del aire en el pulmón; a pesar de ello, los patrones de heterogeneidad observados en los pacientes con SDRA responden de forma diversa a la ventilación con PEEP. El uso de maniobras de reclutamiento (MR) se ha propuesto como tratamiento adjunto a la ventilación mecánica para reexpandir el tejido pulmonar colapsado que se observa en el SDRA. Sin embargo, no está claro que las MR sean útiles cuando los pacientes se ventilan con PEEP elevada o cuando están afectos de fibrosis, alteraciones de la caja torácica o alteraciones de la volemia, entre otras afecciones. De la misma manera, a la hora de tomar una decisión acerca de las MR, hay que considerar no sólo su efectividad a corto o largo plazo y su reversibilidad, sino también que las MR pueden no estar exentas de efectos adversos derivados de las elevadas presiones que se alcanzan. Entre ellos destacan la posibilidad de barotrauma y alteraciones hemodinámicas como la dificultad del retorno venoso y la disminución del volumen sistólico y el flujo aórtico, así como la posibilidad de translocación bacteriana a la circulación sistémica u otros órganos. Este artículo analiza los efectos derivados del uso de MR como tratamiento adyuvante en los pacientes con SDRA, frente a su efectividad y el beneficio potencial, así como las diversas interacciones con diversos trastornos relacionados con el SDRA.

Correspondencia: Dr. L. Blanch.

Centro de Críticos. Hospital de Sabadell. Corporació Parc Taulí.

Parc Taulí, s/n. 08208 Sabadell. Barcelona. España.

Correo electrónico: 1blanch@ tauli.cat
PALABRAS CLAVE: Ventilación mecánica. SDRA. Colapso pulmonar. Reclutamiento alveolar. PEEP. Hemodinámica. Translocación bacteriana.

\section{UTLITY OF RECRUITMENT MANEUVERS (CON)}

A reduction in both total lung volume and in lung parenchyma useful for gas exchange has been observed in ARDS patients. Applying an appropriate ventilatory pattern that includes PEEP can open up collapsed areas of the lung to aeration, thus ensuring a more homogeneous distribution of air in the lung. However, the heterogeneous patterns observed in patients with ARDS vary widely in their response to ventilation with PEEP. Recruitment maneuvers (RM) have been proposed as an adjuvant treatment to mechanical ventilation to re-expand collapsed lung tissue in ARDS. Nevertheless, it is unclear whether RM are useful when patients are ventilated with high PEEP or when they have fibrosis, stiff chest wall, or hypovolemia, among other conditions. Likewise, decisions about RM must take into account not only their short- and long-term efficacy and reversibility, but also possible adverse effects derived from the high pressures reached during $R M$, including barotrauma, hemodynamic alterations, reduced systolic volume and aortic flow, and difficulties in venous return, as well as the possibility of bacterial translocation to the bloodstream or other organs. This article review the effects of RM as adjuvant treatment to mechanical ventilation in ARDS patients and discuss its efficacy and potential benefits as well as the different interactions that RM can have with the diverse conditions that can be associated to ARDS.

KEY WORDS: Mechanical ventilation. ARDS. Lung collapse. Alveolar recruitment. PEEP. Hemodynamics. Bacterial translocation. 


\section{INTRODUCCIÓN}

Las alteraciones fisiopatológicas asociadas al síndrome del distrés respiratorio agudo (SDRA) se caracterizan por alterar la función pulmonar, produciendo edema pulmonar no cardiogénico, disminución de la adaptabilidad del sistema respiratorio, hipoxemia severa y elevación del espacio muerto alveolar y del shunt intrapulmonar. Estudios pioneros en los que se analiza la morfología del pulmón con SDRA mediante tomografía computarizada (TC) han mostrado una evidente reducción del volumen pulmonar, así como una disminución del parénquima pulmonar útil para el intercambio gaseoso, de donde nace el término $b a$ by lung. La aplicación de PEEP restituye el aireamiento de las zonas pulmonares colapsadas o atelectasiadas y disminuye la apertura y el cierre cíclicos de distintas regiones alveolares al incrementar el volumen pulmonar en las zonas con colapso o con tendencia a éste; a su vez, un nivel de PEEP moderado/alto permite una distribución mucho más homogénea del volumen corriente inspiratorio ${ }^{1-4}$. Si el reclutamiento pulmonar es funcional, se restituye la ventilación y la perfusión de las áreas reclutadas y mejoran el intercambio de gases y la mecánica pulmonar, aunque no siempre es así.

Los pacientes con SDRA muestran distintos patrones de heterogeneidad y respuestas muy distintas a la ventilación con presión positiva. En los pacientes con imagen de alteración difusa en la TC, la aplicación de PEEP mejora el reclutamiento alveolar y el intercambio de gases. En los pacientes con alteraciones focales en la TC, la aplicación de PEEP produce sobredistensión de las zonas previamente aireadas, con lo que se incrementa el riesgo de lesión inducida por el ventilador. Por otra parte, las zonas colapsadas pueden airearse parcialmente y experimentar fenómenos cíclicos de apertura y cierre, lo que facilita la lesión, o permanecer colapsadas, en cuyo caso la sobredistensión del resto de parénquima pulmonar todavía reducirá más su tamaño y su volumen ${ }^{2-4}$. Por este motivo no se observan variaciones en el intercambio gaseoso y/o en la mecánica pulmonar. Por lo tanto, la aplicación puntual o sostenida de presiones positivas intratorácicas elevadas a fin de conseguir y mantener un reclutamiento alveolar generalizado está sujeta a controversia ${ }^{5}$

El objetivo del presente artículo es ofrecer una visión de cautela a la hora de aplicar maniobras de reclutamiento alveolar agresivas a pacientes con SDRA describiendo sus efectos adversos y la falta de evidencia científica en estudios en humanos.

\section{EFECTOS ADVERSOS DURANTE EL RECLUTAMIENTO ALVEOLAR AGRESIVO}

Las maniobras de reclutamiento alveolar (MR) producen altas presiones intratorácicas y transpulmonares, lo que puede ocasionar una reducción del gasto cardíaco fundamentalmente por dos mecanismos:

1. La transmisión de la presión de la vía aérea al espacio pleural incrementa la presión intrapleural im- pidiendo el retorno venoso y el llenado del ventrículo derecho.

2. El reclutamiento alveolar se asocia con un «desreclutamiento de la circulación pulmonar» debido a que la distensión pulmonar secundaria al incremento de la presión transpulmonar comprime los pequeños vasos alveolares. En consecuencia, se incrementa la poscarga del ventrículo derecho con disminución consiguiente del volumen sistólico.

Aunque muchos de los estudios iniciales sobre reclutamiento alveolar concluyeron que los cambios hemodinámicos durante la MR están ausentes o son poco importantes ${ }^{6-8}$, en la mayoría de ellos se midieron solamente las presiones intravasculares y no se determinó ni el gasto ni la función cardíaca.

En los últimos años, diversos estudios clínicos y experimentales han mostrado la presencia de efectos cardiovasculares asociados a la $\mathrm{MR}^{9-14}$. El efecto hemodinámico principal encontrado es una disminución significativa del gasto cardíaco durante la realización de la maniobra. Este fenómeno habitualmente es reversible en pocos minutos tras la finalización de la maniobra; sin embargo, puede producirse un descenso del gasto cardíaco de gran magnitud. En este sentido, se han observado caídas de hasta un $60 \%$ del gasto cardíaco que tuvieron como consecuencia un incremento de los fármacos vasoactivos para lograr la estabilización hemodinámica de los pacientes estudiados ${ }^{14}$. Generalmente, los cambios observados en la presión arterial media son menos pronunciados que el descenso en el gasto cardíaco ${ }^{13,14}$, por lo que la monitorización de la presión arterial subestima los verdaderos efectos cardiovasculares de las MR, los cuales podrían ser detectados a través de la monitorización continua del gasto cardíaco.

El grado de deterioro hemodinámico producido por las MR y su impacto clínico depende en gran medida de la volemia en el momento de la maniobra. Odenstedt et $\mathrm{al}^{13}$ demostraron en un modelo porcino que la hipovolemia puede aumentar los efectos hemodinámicos de las MR y que la hipervolemia puede atenuarlos. Estos resultados se han confirmado recientemente en otro estudio experimental ${ }^{12}$, en el que se realizaron las MR en situaciones de normovolemia, hipovolemia e hipervolemia y se calcularon el gasto cardíaco además de medidas ecocardiográficas de diámetros ventrículares, fracción de acortamiento e índice de excentricidad. En este modelo animal de lesión aguda pulmonar, la MR disminuyó significativamente el gasto cardíaco un $89 \%$ en situación de hipovolemia, frente a un 86 y un $56 \%$ en normovolemia e hipervolemia respectivamente. Se observó asimismo una disminución del diámetro telediastólico del ventrículo izquierdo más pronunciada en hipovolemia. Por otro lado, la ecocardiografía realizada durante una MR mostró que el ventrículo derecho fue totalmente comprimido en hipovolemia. Los autores señalan que el mecanismo dominante de deterioro hemodinámico durante la hipovolemia fue la dificultad de retorno venoso y llenado ventricular, mientras que en situaciones de normovolemia e hipervolemia el aumento de la poscarga del ventrículo derecho (re- 
flejado en una disfunción ventricular derecha con incremento del índice de excentricidad y movimiento paradójico septal) fue la causa principal de disminución de gasto cardíaco.

El tipo de maniobra utilizada y la etiología del proceso que causa el daño pulmonar son factores que también influyen en la gravedad de los cambios hemodinámicos asociados a las MR, como demuestran datos recientes ${ }^{10,13}$. Las MR realizadas mediante presión positiva continua en la vía aérea (CPAP) producen descensos más marcados del gasto cardíaco y de la presión arterial que las MR obtenidas con ventilación controlada por presión, probablemente porque ésta proporciona una presión inspiratoria máxima limitada e intermitente en la vía aérea, con lo que mejora el retorno venoso comparado con CPAP. Asimismo, los efectos hemodinámicos fueron más llamativos en el modelo experimental de lesión pulmonar aguda originada por endotoxina, en el que se observó una depresión grave circulatoria con descenso marcado del flujo aórtico $(80 \%)$ y del aporte de oxígeno total, mesentérico y renal, posiblemente secundarios a las complejas alteraciones cardiocirculatorias presentes en la sepsis.

Los efectos deletéreos hemodinámicos de la MR son de corta duración, por lo que podría argumentarse que son aceptables para la seguridad del paciente. Sin embargo, el grado de deterioro hemodinámico, con una combinación de hipertensión pulmonar, hipotensión y disminución del gasto cardíaco puede ser variable, y en un paciente hipovolémico con sepsis podría ocasionar un riesgo vital. Por lo tanto, es preciso conocer las condiciones clínicas y hemodinámicas del paciente escogiendo la MR idónea para garantizar su seguridad y eficacia porque, como dice Jardin ${ }^{15}$, «... incluso si está pobremente aireado, un pulmón dañado con una circulación preservada sobrevivirá. Por el contrario, un pulmón aireado al máximo pero sin ninguna circulación es un órgano inútil...».

El reclutamiento alveolar agresivo a su vez también puede favorecer la translocación bacteriana del pulmón a la circulación sistémica y a otros órganos ${ }^{16}$. En diversos modelos animales basados en la inoculación intratraqueal de bacterias se ha valorado la descompartimentalización de la respuesta local según la modalidad ventilatoria aplicada ${ }^{17}$. Cakar et al ${ }^{18}$, usando la translocación bacteriana como marcador de lesión pulmonar, estudiaron si la aplicación de presión elevada intermitente de manera puntual, no mantenida, como sería el caso de las MR, se asociaba con riesgo de compromiso de la barrera alveolocapilar. A pesar de no obtener resultados concluyentes debido a la limitación temporal inherente al modelo, los autores recomiendan el diseño de estudios que comparen a más largo plazo si las MR se asocian con mayores índices de translocación bacteriana. Por lo tanto, la presencia de infección pulmonar puede ser considerada como una limitación a la hora de decidir si añadir MR como adyuvantes a la ventilación mecánica.

Además de la depresión hemodinámica, en términos de disminución del gasto cardíaco, de disminución de la presión arterial media y de alteraciones en el ín- dice de ventilación/perfusión, se ha demostrado que las MR no sólo no son efectivas en la reducción del daño epitelial secundario al ácido oleico ${ }^{19}$, sino que pueden producir disfunción epitelial y promover el edema ${ }^{20}$.

Más allá de las consecuencias puramente hemodinámicas del reclutamiento alveolar antes consideradas, algunos autores han descrito alteraciones en la circulación esplácnica ${ }^{21}$ y disfunción hepática tras la aplicación de $\mathrm{MR}^{22}$. En este modelo experimental los animales con lesión pulmonar aguda inducida por lavado que habían sido reclutados presentaron una importante respuesta inflamatoria, con reclutamiento de neutrófilos y linfocitos en los sinusoides hepáticos, que estaba acompañada de elevadas concentraciones séricas de enzimas hepáticas y ácido hialurónico ${ }^{22}$, que se mantenían a las $6 \mathrm{~h}$ de la MR. No obstante, no se puede descartar que la disfunción hepática observada fuese secundaria a la alteración en la perfusión en el hígado producida por las elevadas presiones intratorácicas durante el reclutamiento alveolar.

De igual manera no hay que obviar uno de los riesgos más importantes que tener en cuenta ante la decisión de aplicar MR, como es la posibilidad de producir barotrauma. El incremento sostenido o puntual de la presión positiva puede comportar un riesgo implícito de barotrauma o sobredistensión regional, que gana importancia en el caso de heterogeneidad pulmonar. El potencial beneficio en reclutamiento pulmonar depende del nivel de PEEP aplicado tras la MR que impida el desreclutamiento ${ }^{23-25}$ y el ajuste de los niveles de PEEP tras la maniobra de reclutamiento es determinante en la reversibilidad del beneficio aportado.

Una vez valorados de manera integrada los posibles efectos adversos frente a la eficacia temporal de las MR y en el caso de que el reclutamiento alveolar sea imprescindible, algunos autores ${ }^{26}$ han propuesto el empleo de las MR selectivas o con presión moderada como intervención que no comporta alteración hemodinámica en modelos de lesión pulmonar aguda en cerdos con atelectasia lobar. Su efectividad se evaluó mediante tomografía por impedancia eléctrica, y se describen resultados positivos alcanzando reclutamiento a una menor presión que no comporta depresión circulatoria ${ }^{26-28}$. Sin embargo, esta respuesta podría estar muy condicionada por el modelo experimental de lesión pulmonar utilizado ${ }^{29}$.

\section{ESTUDIOS CLÍNICOS SOBRE ESTRATEGIAS DE VENTILACIÓN PROTECTORA ASOCIADA O NO A RECLUTAMIENTO AGRESIVO}

El empleo de un volumen corriente bajo ha ganado una enorme aceptación ${ }^{30}$ después de los resultados de los últimos estudios aleatorizados en una gran población de pacientes con SDRA ${ }^{31-36}$. Asimismo, el análisis de los parámetros ventilatorios y de las presiones resultantes de su aplicación indica que la presión elevada también es deletérea. De hecho, en los 3 estu$\operatorname{dios}^{31,35,37}$ donde se muestra una reducción de la mortalidad, la presión meseta del grupo control es generalmente $<30 \mathrm{cmH}_{2} \mathrm{O}$. Este hecho ha llevado a 
postular que la presión meseta debe limitarse también y se considera seguro que sea $<32 \mathrm{cmH}_{2} \mathrm{O}$. Estudios fisiológicos recientes ${ }^{38}$ han demostrado que los pacientes ventilados con estrategia de ventilación protectora (ARDSnet) que presentaban presiones meseta $>28 \mathrm{cmH}_{2} \mathrm{O}$ mostraban mayores áreas de sobredistensión pulmonar, en el análisis morfológico del pulmón mediante TC, y presencia de citocinas proinflamatorias en plasma. Paralelamente a estos hallazgos, otro grupo de investigadores ${ }^{39}$ describió que un patrón de concavidad en la representación gráfica de la onda de presión de la vía aérea en función del tiempo durante el periodo de flujo aéreo constante no sólo era indicio de sobredistensión pulmonar, sino que también se asociaba a un patrón proinflamatorio en el plasma de estos pacientes.

La aplicación de PEEP elevada y/o de maniobras de reclutamiento alveolar dirigidas a maximizar la apertura y posterior ventilación de unidades colapsadas está sujeta a controversia. El estudio ALVEOLI ${ }^{36}$ no demostró una mayor eficacia del empleo de PEEP elevada y volumen corriente (VT) de $6 \mathrm{ml} / \mathrm{kg}$ al compararlo con la estrategia utilizada en el estudio ARMA $^{35}$ (PEEP moderada y VT de $6 \mathrm{ml} / \mathrm{kg}$ ) en pacientes con SDRA. Posteriormente, Meade et $\mathrm{al}^{40} \mathrm{y}$ Mercat et $\mathrm{al}^{41}$ en estudios aleatorizados en pacientes con ALI/ARDS y ventilados con VT de $6 \mathrm{ml} / \mathrm{kg}$, analizaron si la aplicación de PEEP elevada, permitiendo presiones meseta de hasta $40 \mathrm{cmH}_{2} \mathrm{O}$ el primero y utilizando un nivel de PEEP a fin de conseguir una presión meseta de hasta $28 \mathrm{cmH}_{2} \mathrm{O}$ el segundo en los grupos tratamiento, no demostraron mejoras en términos de supervivencia al compararlos con los grupos controles que recibieron un nivel de PEEP menor. Sin embargo, Meade et $\mathrm{al}^{40}$ y Mercat et $\mathrm{al}^{41}$ encontraron que la PEEP elevada se asoció a menos episodios de hipoxemia severa y a un menor requerimiento de terapias de rescate. Con base en estos amplios estudios, debería recomendarse la utilización de un VT bajo y de un nivel de PEEP entre moderado y alto, cuya presión meseta resultante no supere los $30 \mathrm{cmH}_{2} \mathrm{O}$.

El empleo de maniobras de reclutamiento con la finalidad de obtener un reclutamiento máximo y a partir de este aplicar un nivel de PEEP óptimo capaz de mantener $\mathrm{PaO}_{2} / \mathrm{FiO}_{2}$ elevadas sólo se ha demostrado útil en estudios experimentales de modelos de SDRA diferentes del síndrome observado en humanos. En la actualidad sólo contamos con estudios que ofrecen resultados no concluyentes en variables fisiológicas y a corto plazo. Ningún estudio en humanos ha analizado posibles efectos secundarios de las maniobras de reclutamiento a medio o largo plazo. Finalmente, la escasa evidencia científica actual no avala el uso sistemático de las MR en todos los pacientes con ALI/ARDS. Ozcenski et al $^{42}$ aleatorizó a pacientes con SDRA que eran ventilados con un nivel óptimo de PEEP a recibir MR o un simulacro, y no encontraron alteraciones significativas en el intercambio gaseoso o en la mecánica pulmonar. Finalmente, cuando se aplicaron MR en el grupo de PEEP elevada en el estudio ALVEOLI ${ }^{43}$ y en el grupo de «open lung ventilation» en el trabajo de Meade et $\mathrm{al}^{40}$, no sólo no se observó mejoría en los parámetros fisiológicos habituales, sino que las MR se asociaron a complicaciones en un elevado número de pacientes, algunas de ellas importantes.

\section{CONCLUSIONES}

El reclutamiento alveolar agresivo en pacientes con SDRA no está exento de riesgos y a su vez los beneficios de su uso no están absolutamente demostrados. Los estudios actuales únicamente muestran mejoras en la oxigenación en grupos de pacientes ventilados inicialmente con PEEP moderada o muy baja y no hay datos sobre los pacientes no respondedores. Tampoco hay evidencia que permita recomendar qué técnica utilizar a la hora de reclutar, ni tampoco cuál es a priori el paciente que va a beneficiarse por incrementarse la oxigenación y disminuirse el espacio muerto alveolar, con las dudas de si esta medida será determinante para el pronóstico final del paciente. La medicina intensiva no es únicamente aplicar el mejor y más contrastado conocimiento al paciente crítico, sino en no añadir más daño al ya existente. La información científica actual sobre las maniobras de reclutamiento alveolar aplicadas en cualquier momento del proceso de un paciente con SDRA no permite en absoluto confirmar este segundo punto. Por ello, debemos concluir que no hay evidencia científica que aconseje el empleo sistemático de las RM para optimizar la PEEP y mejorar el pronóstico de los pacientes con SDRA.

\section{AGRADECIMIENTOS}

Josefina Lopez-Aguilar está adscrita al Programa de Estabilización de Investigadores en el Sistema Nacional de Salud (I3SNS) ISCIII-Generalitat de Catalunya.

\section{BIBLIOGRAFÍA}

1. Gattinoni L, D’Andrea L, Pelosi P, Vitale G, Pesenti A, Fumagalli R. Regional effects and mechanism of positive endexpiratory pressure in early adult respiratory distress syndrome. JAMA. 1993;269:2122-7.

2. Gattinoni L, Pelosi P, Crotti S, Valenza F. Effects of positive end-expiratory pressure on regional distribution of tidal volume and recruitment in adult respiratory distress syndrome. Am J Respir Crit Care Med. 1995;151:1807-14.

3. Vieira SR, Puybasset L, Richecoeur J, Lu Q, Cluzel P, Gusman $\mathrm{PB}$, et al. A lung computed tomographic assessment of positive end-expiratory pressure-induced lung overdistension. Am J Respir Crit Care Med. 1998; 158:1571-7.

4. Rouby JJ, Puybasset L, Nieszkowska A, Lu Q. Acute respiratory distress syndrome: lessons from computed tomography of the whole lung. Crit Care Med. 2003;31:S285-95.

5. Gattinoni L, Caironi P, Cressoni M, Chiumello D, Ranieri VM, Quintel M, et al. Lung recruitment in patients with the acute respiratory distress syndrome. N Engl J Med. 2006;354:1775-86.

6. Lapinsky SE, Aubin M, Mehta S, et al. Safety and efficacy of a sustained inflation for alveolar recruitment in adults with respiratory failure. Intensive Care Med. 1999;25:1297-301.

7. Fujino Y, Goddon S, Dolhnikoff M, Hess D, Amato MB, Kacmarek RM. Repetitive high-pressure recruitment maneuvers required to maximally recruit lung in a sheep model of acute respiratory distress syndrome. Crit Care Med. 2001;29:1579-86.

8. Villagrá A, Ochagavía A, Vatua S, Murias G, Del Mar Fernández M, Lopez Aguilar J et al. Recruitment maneuvers du- 
ring lung protective ventilation in acute respiratory distress syndrome. Am J Respir Crit Care Med. 2002;165:165-70.

9. Celebi S, Köner O, Menda F, Korkut K, Suzer K, Cakar N. The pulmonary and hemodynamic effects of two different recruitment maneuvers after cardiac surgery. Anesth Analg. 2007;104:384-90.

10. Lim SC, Adams AB, Simonson DA, Dries DJ, Broccard AF, Hotchkiss JR, et al. Transient hemodynamic effects of recruitment maneuvers in three experimental models of acute lung injury. Crit Care Med. 2004;32:2378-84.

11. Nielsen J, Østergaard M, Kjaergaard J, Tingleff J, Berthelsen PG, Nygård E, et al. Lung recruitment maneuver depresses central hemodynamics in patients following cardiac surgery. Intensive Care Med. 2005;31:1189-94.

12. Nielsen J, Nilsson M, Fredén F, Hultman J, Alström U, Kjaergaard J, et al. Central hemodynamics during lung recruitment maneuvers at hypovolemia, normovolemia and hypervolemia. A study by echocardiography and continous pulmonary artery flow measurements in lung-injured pigs. Intensive Care Med. 2006;32:585-94.

13. Odenstedt H, Aneman A, Kárason S, Stenqvist O, Lundin $\mathrm{S}$. Acute hemodynamic changes during lung recruitment in lavage and endotoxin-induced ALI. Intensive Care Med. 2005;31: $112-20$.

14. Toth I, Leiner T, Mikor A, Szakmany T, Bogar L, Molnar Z. Hemodynamic and respiratory changes during lung recruitment and descending optimal positive end-expiratory pressure titration in patients with acute respiratory distress syndrome. Crit Care Med. 2007;35:787-93.

15. Jardin F. Acute leftward septal shift by lung recruitment maneuver. Intensive Care Med. 2005;31:1148-9.

16. Lachmann RA, Van Kaam AH, Haitsma JJ, Lachmann B. High positive end-expiratory pressure levels promote bacterial translocation in experimental pneumonia. Intensive Care Med. 2007:33:1800-4.

17. Ozcan PE, Cakar N, Tugrul S, Akinci O, Cagatay A, Yilmazbayhan D, et al. The effects of airway pressure and inspiratory time on bacterial translocation. Anesth Analg. 2007;104: 391-6.

18. Cakar N, Akinci O, Tugrul S, Ozcan PE, Esen F, Eraksoy H, et al. Recruitment maneuver: does it promote bacterial translocation? Crit Care Med. 2002;30:2103-6.

19. Frank JA, McAuley DF, Gutierrez JA, Daniel BM, Dobbs L, Matthay MA. Differential effects of sustained inflation recruitment maneuvers on alveolar epithelial and lung endothelial injury. Crit Care Med. 2005;33:181-8.

20. Constantin JM, Cayot-Constantin S, Roszyk L, Futier E, Sapin V, Dastugue B, et al. Response to recruitment maneuver influences net alveolar fluid clearance in acute respiratory distress syndrome. Anesthesiology. 2007;106:944-51.

21. Nunes S, Rothen HU, Brander L, Takala J, Jakob SM. Changes in splanchnic circulation during an alveolar recruitment maneuver in healthy porcine lungs. Anesth Analg. 2004;98: $1432-8$.

22. Kredel M, Muellenbach RM, Brock RW, Wilckens HH, Brederlau J, Roewer N, et al. Liver dysfunction after lung recruitment manoeuvres during pressure-controlled ventilation in experimental acute respiratory distress. Crit Care. 2007;11:R13.

23. Cakar N, Der Kloot TV, Youngblood M, Adams A, Nahum A. Oxygenation response to a recruitment maneuver during supine and prone positions in an oleic acid-induced lung injury model. Am J Respir Crit Care Med. 2000;161:1949-56.

24. Pelosi P, Goldner M, McKibben A, Adams A, Eccher G, Caironi P, et al. Recruitment and derecruitment during acute respiratory failure: an experimental study. Am J Respir Crit Care Med. 2001;164:122-30.

25. Piacentini E, Villagrá A, López-Aguilar J, Blanch L. Clinical review: the implications of experimental and clinical studies of recruitment maneuvers in acute lung injury. Crit Care. 2004;8: $115-21$.

26. Hansen LK, Sloth E, Nielsen J, Koefoed-Nielsen J, Lambert $\mathrm{P}$, Lunde S, et al. Selective recruitment maneuvers for lobar atelectasis: effects on lung function and central hemodynamics: an experimental study in pigs. Anesth Analg. 2006;102:1504-10.

27. Hansen LK, Koefoed-Nielsen J, Nielsen J, Larsson A. Are selective lung recruitment meneuvers hemodynamically safe in severe hypovolemia? An experimental study in hypovolemic pigs with lobar collapse. Anesth Analg. 2007:105:729-34.

28. Odenstedt H, Lindgren S, Olegård C, Erlandsson K, Lethvall $\mathrm{S}$, Aneman A, et al. Slow moderate pressure recruitment maneuver minimizes negative circulatory and lung mechanic side effects: evaluation of recruitment maneuvers using electric impedance tomography. Intensive Care Med. 2005;31:1706-14.

29. Kloot TE, Blanch L, Melynne Youngblood A, Weinert C, Adams AB, Marini JJ, et al. Recruitment maneuvers in three experimental models of acute lung injury. Effect on lung volume and gas exchange. Am J Respir Crit Care Med. 2000;161:1485-94.

30. Esteban A, Ferguson ND, Meade MO, Frutos-Vivar F, Apezteguia C, Brochard L, et al; VENTILA Group. Evolution of mechanical ventilation in response to clinical research. Am J Respir Crit Care Med. 2008;177:170-7.

31. Amato MBP, Barbas CSV, Medeiros DM, Magaldi RB, Schettino GP, Lorenzi-Filho G, et al. Effect of a protective-ventilation strategy on mortality in the acute respiratory distress syndrome. N Engl J Med. 1998;338:347-54.

32. Stewart TE, Meade MO, Cook DJ, Granton JT, Hodder RV, Lapinsky SE, et al. Evaluation of a ventilation strategy to prevent barotrauma in patients at high risk for acute respiratory distress syndrome. N Engl J Med. 1998;338:355-61.

33. Brochard L, Roudot F, Roupie E, Delclaux C, Chastre J, Fernández E, et al. Tidal volume reduction for prevention of ventilator induced lung injury in the acute respiratory distress syndrome. Am J Respir Crit Care Med. 1998;158:1831-8.

34. Brower RG, Shanholtz CB, Fessler HE, Shade DM, White P $\mathrm{Jr}$, Wiener CM, et al. Prospective, randomized, controlled clinical trial comparingtraditional versus reduced tidal volume ventilation in acute respiratory distress syndrome. Crit Care Med. 1999;27:1492-8.

35. The Acute Respiratory Distress Syndrome Network. Ventilation with lower tidal volumes as compared with traditional tidal volumes for acute lung injury and the acute respiratory distress syndrome. N Engl J Med 2000;342:1301-8.

36. Brower RG, Lanken PN, MacIntyre N, Matthay MA, Morris A, Ancukiewicz M, et al; National Heart, Lung Blood Institute ARDS Clinical Trials Network. Higher versus lower positive endexpiratory pressures in patients with the acute respiratory distress syndrome. N Engl J Med. 2004;351:327-36.

37. Villar J, Kacmarek RM, Pérez-Méndez L, Aguirre-Jaime A. A high positive end-expiratory pressure, low tidal volume ventilatory strategy improves outcome in persistent acute respiratory distress syndrome: a randomized, controlled trial. Crit Care Med. 2006;34:1311-8

38. Terragni PP, Rosboch G, Tealdi A, Corno E, Menaldo E, Davini $\mathrm{O}$, et al. Tidal hyperinflation during low tidal volume ventilation in acute respiratory distress syndrome. Am J Respir Crit Care Med. 2007;175:160-6.

39. Grasso S, Stripoli T, De Michele M, Bruno F, Moschetta M, Angelelli G, et al. ARDSnet ventilatory protocol and alveolar hyperinflation: role of positive end-expiratory pressure. Am J Respir Crit Care Med. 2007;176:761-7.

40. Meade MO, Cook DJ, Guyatt GH, Slutsky AS, Arabi YM, Cooper DJ, et al; Lung Open Ventilation Study Investigators. Ventilation strategy using low tidal volumes, recruitment maneuvers, and high positive end-expiratory pressure for acute lung injury and acute respiratory distress syndrome: a randomized controlled trial. JAMA. 2008;299:637-45.

41. Mercat A, Richard JC, Vielle B, Jaber S, Osman D, Diehl JL, et al; Expiratory Pressure (Express) Study Group. Positive end-expiratory pressure setting in adults with acute lung injury and acute respiratory distress syndrome: a randomized controlled trial. JAMA. 2008;299:646-55.

42. Oczenski W, Hörmann C, Keller C, Lorenzl N, Kepka A, Schwarz S, et al. Recruitment maneuvers after a positive end-expiratory pressure trial do not induce sustained effects in early adult respiratory distress syndrome. Anesthesiology. 2004;101:620-5.

43. Brower RG, Morris A, MacIntyre N, Matthay MA, Hayden D, Thompson T, et al. ARDS Clinical Trials Network, National Heart, Lung, and Blood Institute, National Institutes of Health. Effects of recruitment maneuvers in patients with acute lung injury and acute respiratory distress syndrome ventilated with high positive end-expiratory pressure. Crit Care Med. 2003;31:2592-7. 